\title{
Diurnal rhythm of free estradiol during the menstrual cycle
}

\author{
Ai-Min Bao ${ }^{1,2}$, Rong-Yu Liu ${ }^{2}$, Eus J W van Someren ${ }^{3,5}$, Michel A Hofman ${ }^{3}$, Yun-Xia Cao ${ }^{4}$ and Jiang-Ning Zhou ${ }^{1,2}$ \\ ${ }^{1}$ Department of Neurobiology, School of Life Science, University of Science and Technology of China, Hefei 230032, Anhui, \\ People's Republic of China, ${ }^{2}$ Anhui Geriatrics Institute. The First Affiliated Hospital of Anhui Medical University. Hefei 230022, Anhui, \\ People's Republic of China, ${ }^{3}$ Netherlands Institute for Brain Research, 1105 AZ Amsterdam ZO, The Netherlands, \\ ${ }^{4}$ Department of Obstetrics and Gynecology. The First Affiliated Hospital of Anhui Medical University, Hefei 230022, Anhui, People's Republic of China \\ and ${ }^{5}$ Netherlands Institute for Clinical and Experimental Neuroscience, VU University Medical Centre, 1105 AZ Amsterdam ZO, The Netherlands \\ (Correspondence should be addressed to Jiang-Ning Zhou, Anhui Geriatrics Institute, The First Affiliated Hospital of Anhui Medical University, Hefei \\ 230022, Anhui, People's Republic of China; Email: anhuigi@mail.hf.ah.cn)
}

\begin{abstract}
Objective: To investigate the diurnal rhythm of estrogens in normally cyclic women during reproductive life.

Design: Multiple saliva sampling in normally cyclic healthy women during reproductive life at different phases of their menstrual cycles was carried out.

Methods: Salivary estradiol was measured by radioimmunoassay in samples collected every $2 \mathrm{~h}$ for $24 \mathrm{~h}$ from 15 normally cyclic healthy women during reproductive life during the menstrual phase, the late follicular/peri-ovulation phase, the early to mid luteal phase and the late luteal phase, respectively, of their menstrual cycles. The levels of salivary estradiol were analyzed by means of periodic regression.

Results: A daily biological rhythm of free estradiol was found after quantification with a nonlinear periodic regression model. The observed diurnal free estradiol rhythm consists of two major components: an asymmetrically peaked diurnal cycle and ultradian harmonics in the range of 6 to $12 \mathrm{~h}$. The diurnal and ultradian rhythms were remarkably consistent throughout the menstrual cycle in terms of mesor ( $24 \mathrm{~h}$ mean level), peak width and amplitude. There was a tendency for the 24-h rhythm acrophases to converge in the early morning, while the acrophase of the menstrual phase occurred significantly later than in the late follicular/peri-ovulation phase.

Conclusions: The diurnal rhythm of estradiol has a similar complex temporal organization for different menstrual phases. The menstrual cycle mainly modulates the acrophase of the diurnal rhythm.
\end{abstract}

European Journal of Endocrinology 148 227-232

\section{Introduction}

It is known that estrogens are more than just 'sex hormones' that play a principal role in regulating behavioral and physiological events essential for successful procreation (1). Estrogens are also important for the protection of the cardiovascular system (2) and for the maintenance of bone $(3,4)$. Estrogens play an important role in the sexual differentiation of the brain (5) and in modulating the regulation of the autonomic and reproductive neuroendocrine system, mood, and cognition (5-7). Changes in estrogen level in different physiological and pathological situations are a topic of considerable research interest. Previous studies on estrogen levels mainly focused on their fluctuation during the menstrual cycle, postpartum and menopause, using single or multiple time-point samples $(8-10)$. Although studies on estrogen secretion in early puberty, gestation and breast cancer have assumed for a long time that estrogen levels in humans have a diurnal rhythm (11-15), little is known about the fluctuation of estrogens in normally cyclic women over the 24-h period, i.e. its daily biological rhythm. Knowledge of the daily secretion profiles of estrogen will contribute not only to our understanding of its function but also offer useful reference data for further study on the roles of rhythms in estrogen secretion in various diseases.

It is known that both the hypothalamic gonadotropinreleasing hormone $(\mathrm{GnRH})$, and the pituitary luteinizing and follicle-stimulating hormones have pulsatile secretion profiles $(11-16)$ and that these hormones affect the secretion of estrogens within the human hypothalamic-pituitary-gonadal axis. It is suggested in studies with both animal and human material that the hypothalamic suprachiasmatic nucleus (SCN), the endogenous circadian pacemaker in the brain, is sex hormone sensitive and is involved in the generation and regulation of the female reproductive cycle $(17,18)$. Based on the observations mentioned above, 
it is reasonable to hypothesize that the biological rhythm of estrogens is composed of a complex of cycles including not only the monthly cycle but also circadian rhythms and ultradian pulses. The recent development of noninvasive saliva measurements of steroid hormones (19) allowed us to investigate the diurnal and ultradian rhythms of salivary free estradiol, i.e. the biologically active form of estrogen, in women of reproductive age in their natural environment. We further explored whether circadian and ultradian rhythms of free estradiol are modulated by the menstrual cycle.

\section{Materials and methods}

\section{Participants}

Fifteen healthy Chinese women (mean age 32.8 years, range 25-40 years) entered the study between 25 March and 20 June 2001. They all took part on a voluntary basis, and had regular menstrual cycles with periods from 27 to 30 days. Exclusion criteria were as follows: abnormal body mass index (BMI $\leq 18$ or $\geq 25$ ), medication for chronic illnesses, oral contraceptives within the last 6 months, lactating within the last 12 months, evidence of infertility, or presence of sexually transmitted diseases. All subjects signed a consent form and the study was approved by the Anhui Medical University review board.

\section{Procedures}

Subjects participated in the study for a total of four days over a period of one month, based upon their selfreported menstrual cycle day-count: phase I, one day from day 2-3 (menstrual phase); phase II, one day from day 12-14 (late follicular, peri-ovulation phase); phase III, one day from day 18-23 (early to mid luteal phase); phase IV, one day from day 25-28 (late luteal phase). In phase II, ovulation was determined by changes in body basal temperature and every subject had an ovary ultrasonic examination in the $B$ mode to help ensure the phase according to the dimension of the dominant follicle and to ensure the presence of a normal genital system (20). At the entrance time of each day, they were given a package containing 12 Salivettes (Sarstedt, Nümbrecht, Germany) (21) and were asked to collect saliva every $2 \mathrm{~h}$ from $0800 \mathrm{~h}$ to $0600 \mathrm{~h}$ the next morning. Between $2200 \mathrm{~h}$ and $0600 \mathrm{~h}$ the next morning the subjects went to bed/got up with the light(s) turned off/on respectively. This schedule was not significantly different from their work and rest routine. Saliva samples were collected with small polyester tampons held in the mouth for about $5 \mathrm{~min}$ to soak up the saliva and were then placed in the Salivette container. The Salivettes were sealed and stored at $4{ }^{\circ} \mathrm{C}$ in a refrigerator immediately after collection of the samples during the daytime and placed in a cold box beside the subject's pillow at night until the end of each $24-\mathrm{h}$ period. The samples were centrifuged for $10 \mathrm{~min}$ at $3000 \boldsymbol{g}$ and frozen at $-20{ }^{\circ} \mathrm{C}$ until assayed. The subjects were non-heavy exercisers, non-alcohol/noncaffeine drinkers and non-smokers. Subjects were studied in their habitual environment with their habitual occupations. They were advized to brush their teeth without toothpaste, to drink plenty of water but to avoid eating and to rinse their mouths thoroughly with water $30 \mathrm{~min}$ before sampling during the daytime. They were also asked to avoid sexual intercourse during the sampling day. A total of 57 sets of data points were gathered in the present study (no data were available for phase I of subject \#10 and phases I and IV of subject \#8 because of the lack of sufficient saliva at some timepoints). Please note that we did a pilot study before the formal study with two subjects for two days, every other day of phase II (late follicular, peri-ovulation phase) respectively, in which the saliva sampling interval was set at $1 \mathrm{~h}$ between $0800 \mathrm{~h}$ and $2200 \mathrm{~h}$ and at $2 \mathrm{~h}$ between $2200 \mathrm{~h}$ and $0600 \mathrm{~h}$ the next morning. The study of the secretion pattern of estradiol in the pilot study suggested that a 2-h sampling interval throughout the day (which is more convenient for participants) would not affect the deduced diurnal rhythm pattern, which is the main topic of our study.

\section{Salivary estradiol assay}

Salivary estradiol $\left(\mathrm{E}_{2}\right)$ concentrations were measured with the use of a double-antibody radioimmunoassay (RIA) with ${ }^{125}$ I-labeled $E_{2}$. Antibody and tracers were derived from DSL-39100 estradiol (third generation; Diagnostic Systems Laboratories, Inc., Webster, TX, USA). The estradiol antibody is rabbit anti-estradiol in a protein-based buffer with sodium azide. The antibody cross-reacts $6.9 \%$ with estrone and less than $1.0 \%$ with $17 \beta$-estradiol-3-glucuronide. The protocol used was that according to Shirtcliff et al. (22). The standard curve of the salivary estradiol assay was highly reproducible, with an average correlation coefficient of 0.9969 in this study. The average intra- and interassay coefficients of variation were 7.2 and $9.9 \%$ respectively. Analytical recovery was on average $100.7 \%$. Linearity was assessed (1:2 to $1: 12$ dilutions) across the range of measurements with an average recovery of $89 \%$ (range $76 \%-103 \%$ ). The range of standards was $0.375-7.500 \mathrm{pg} / \mathrm{ml}$. The analytic sensitivity was $0.250 \mathrm{pg} / \mathrm{ml}$.

\section{Statistical analysis}

Examination of the raw data indicated that both ultradian and diurnal components were clearly present, with amplitudes of comparable magnitude. Given the variance resulting from such ultradian periods, fitting just a plain 24-h periodic function (cosinor, see reference 23) will only occasionally result in a fit that is 
significantly better than the single parameter model of just a mean with residual error. We used Rao's F-distributed T-statistic (24), which applies a penalty for increasing numbers of parameters, to evaluate several multi-harmonic models and to select the best and most parsimonious model. Applying Nyquist's rule, our sampling frequency allowed for investigation of ultradian rhythms with periods of $5 \mathrm{~h}$ or more. Moreover, skewness or peakedness of circadian physiology is the rule rather than the exception, e.g. the $8 \mathrm{~h}$ to $16 \mathrm{~h}$ asymmetric sleep-wake cycle. Such asymmetric phenomena are by far the most parsimoniously modeled by the peaked and skewed functions proposed by Batschelet (25). Curve fitting was performed using constrained nonlinear regression analysis (SPSS Release 10.0).

After selection of the best model, the possibility of modulation of the parameters by the phase of the menstrual cycle was examined using repeated measures ANOVAs, and circular statistics in the case of parameters representing the acrophase (Jupp's Phi and S for mean angle and angular standard deviation, Mardia-Watson-Wheeler's Chi squared test for evaluation of acrophase differences over the menstrual cycle) (25).

\section{Results}

The best-fitting model describing the rhythmic phenomena, though still parsimonious, was selected from a series of peaked and skewed cosine functions with the addition of a single higher order harmonic, i.e. the 2nd, 3rd or 4 th harmonic (24). The most adequate model turned out to be a peaked cosine function with one additional harmonic, which was significant at $P<0.05$ or better in 42 cases, and at $P<0.10$ or better in 53 cases:

$$
\begin{aligned}
\mathrm{y}[\mathrm{t}]= & M+A * \cos \left[\left(\mathrm{t}-\Phi_{1}\right)+v * \operatorname{SIN}\left(\mathrm{t}-\Phi_{1}\right)\right] \\
& +B * \cos \left(u * \mathrm{t}-\Phi_{2}\right)
\end{aligned}
$$

where $t$ represents the time of day (in radians), $y$ the predicted value of estradiol at time $t$ and $M$ the mesor (24-h mean level). A correction to the mesor is applied in order to account for the asymmetry of the rhythm (25). $A$ is the amplitude of the diurnal component, $\Phi_{1}$ its phase angle and $v$ its parameter of 'peakedness'. The peakedness parameter can be transformed to represent duration in hours (25). B represents the amplitude of the ultradian component, $\Phi_{2}$ its phase angle and $u$ the harmonic of the ultradian component, where $u=2,3$ or 4 correspond with rhythms of 12,8 or $6 \mathrm{~h}$. The acrophase is the corresponding time-point of the predicted highest value of $\mathrm{y}[\mathrm{t}]$. Figure 1 shows an example (subject \#11, phase III) of raw data and the fitted model. All parameters measured over the four phases for which the significance level of the fitted curve was less than 0.10 were used for further calculation of parameter means and examination of modulation of the rhythm throughout the menstrual cycle.

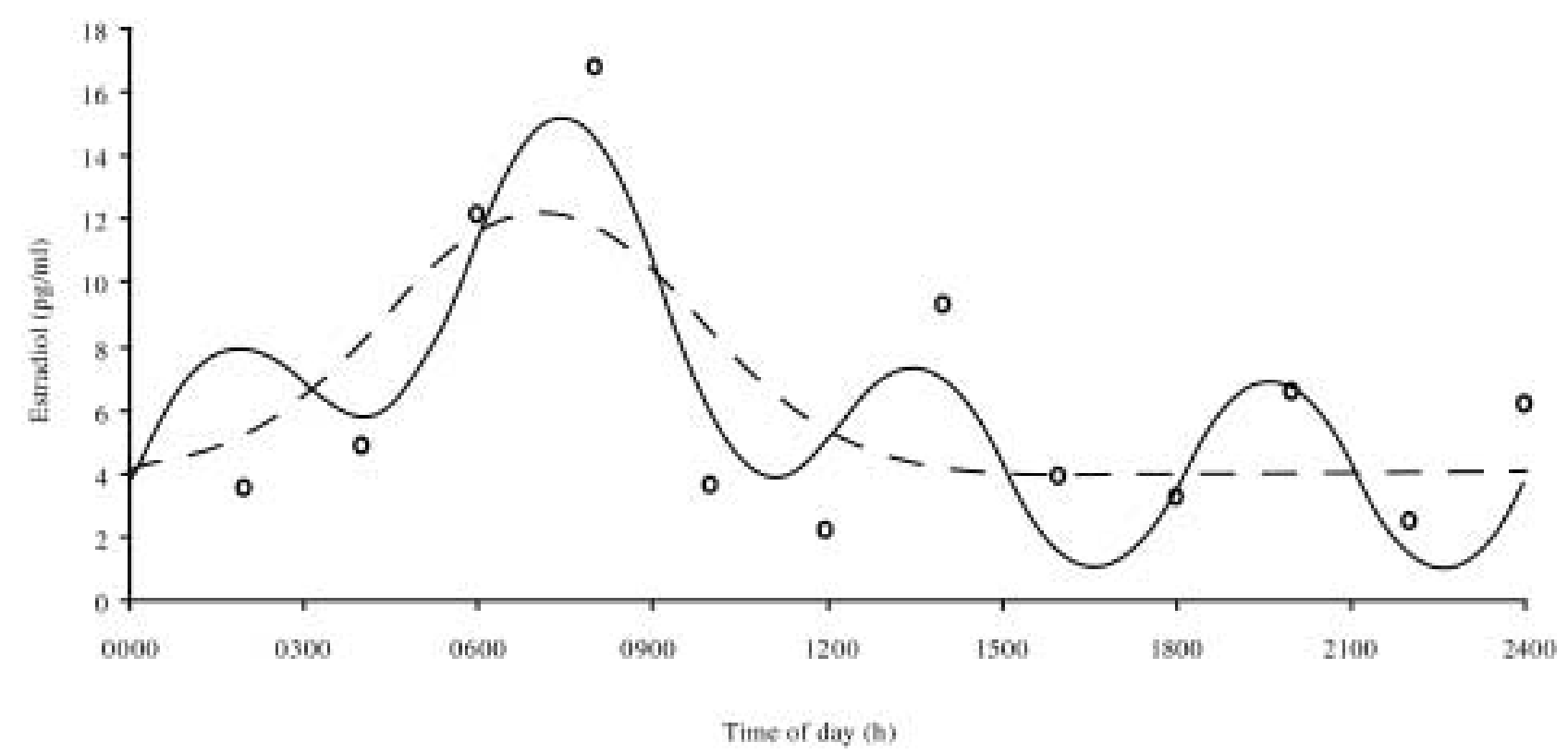

Figure 1 Representative example (subject no.11, phase III) of raw data points (circles), the peaked cosine 24-h rhythmic component (dashed line) and full model including a 4th harmonic (solid line). Note the asymmetry between the peak (0612 $\mathrm{h}$ ) and trough (1748 $\mathrm{h}$ ) duration and the comparable magnitude of the diurnal $(4.2 \mathrm{pg} / \mathrm{ml})$ and ultradian $(3.0 \mathrm{pg} / \mathrm{ml})$ amplitudes. The diurnal acrophase occurred at $0702 \mathrm{~h}$ in the morning. The model accounted for $73 \%$ of the estradiol variance, and was statistically significant $(\mathrm{F}(1,6)=16.35$, $P<0.01$ ). 
The rhythms of free estradiol are remarkably robust throughout the menstrual cycle. With the exception of the acrophase, none of the diurnal and ultradian parameters were affected by the phase of the menstrual cycle. Table 1 shows the means and standard deviations of all relevant parameters, where the mesor and peakedness are expressed in their corrected format (25). The mesors and the amplitudes of the circadian and ultradian components of free estradiol in the menstrual (phase I) and late luteal phases (phase IV) were slightly, but not significantly, lower than those in the late follicular/peri-ovulation (phase II) and early to mid luteal (phase III) phases. The acrophase, however, occurred later in phase I (menstrual phase) of the menstrual cycle. The Mardia-Watson-Wheeler test indicated a significant difference between the acrophase in menstrual phases I and II (Chi squared test $=6.66$, degrees of freedom $(\mathrm{df})=2, P<0.05)$, and a trend towards a difference between the acrophase in menstrual phases I and IV (Chi squared test $=4.92, \mathrm{df}=2, P<0.10$ ). Also, acrophases were found to be concentrated in the early morning (Fig. 2). The most frequently occurring ultradian component, in $56 \%$ of the cases, was the 4 th harmonic, corresponding to a period of $6 \mathrm{~h}$. However, in $23 \%$ of the cases the ultradian rhythm was better described by the third harmonic, corresponding to a period of $8 \mathrm{~h}$, and in $21 \%$ of the cases by the second harmonic, corresponding to a period of $12 \mathrm{~h}$.

\section{Discussion}

The present results indicate that salivary estradiol in women of reproductive age has a nonlinear periodic rhythm throughout the menstrual cycle. This rhythm contains diurnal and ultradian components of similar magnitude. The diurnal component is asymmetrical, i.e. the duration of the peak differs markedly from that of the trough. There is considerable variation among subjects in the ratio between peak and trough duration: both peaked and flat-topped rhythms occur. The rhythms are remarkably consistent throughout the menstrual cycle. The menstrual cycle mainly modulated the acrophase of the estradiol rhythm, indicating that during the menstrual phase the peak levels occurred later in the morning.

There are several protocols designed to study a daily biological rhythm, such as the free-run, the forced desynchrony and the constant-routine protocol (26, $27)$, yet the control methods might not reveal all factors of importance for the biological rhythms of hormones in women. For example, a group of women living together in a room might have 'menstrual synchrony' (28), while synchronization of life style might result in obvious changes in the mood and even in the rhythm of healthy women. In the present study, the subjects were living their normal lives and were tested in the same season and with fixed bedtimes and get-up times, allowing us to investigate the diurnal rhythms of hormones in females living under natural conditions.

Many clinical investigations have demonstrated that salivary steroid measurements are a practical and convenient approach to assess fluctuations in diurnal rhythms. Salivary levels of estradiol are believed to represent accurately the 'free', i.e. the biologically active, fraction in the general circulation $(19,29)$. It has been shown that unconjugated steroids enter the saliva by diffusing through the cells of the salivary glands and that their concentration in saliva does not depend on the rate of saliva production (30). The levels of salivary estradiol in the present study were largely comparable to those in previous studies measured during different menstrual phases $(31,32)$ and as measured in blood samples (20). The difference in free estradiol mesors between the menstrual phases, however, was not statistically significant. This might, on the one hand, be due to between-subject variability of the peak time within the menstrual cycle: both the mesor and the amplitude were elevated in phases II and III as compared with phases I and IV. Moreover, it may be that the previously reported increase in estradiol in phases II and III might be more pronounced in bound estradiol, whereas in the present study we assessed free

Table 1 Characteristics of the diurnal and ultradian rhythms in estradiol throughout the four phases of the menstrual cycle. Results are means \pm standard deviations, but the acrophases are calculated as angular means and standard deviations. The unit for mesor and amplitude is $\mathrm{pg} / \mathrm{ml}$ and the unit for acrophase and peak width is $\mathrm{h}$ and $\mathrm{min}$. The ultradian harmonic row gives the mean and standard deviations of the best fitting harmonic number (of 2,3 or 4 ).

\begin{tabular}{lccc}
\hline Parameter & Phase I & Phase II & Phase III \\
\hline True mesor & $4.93 \pm 1.27$ & $6.07 \pm 2.45$ & $5.79 \pm 2.46$ \\
24-h amplitude & $3.14 \pm 1.60$ & $5.15 \pm 4.22$ & $4.66 \pm 3.18$ \\
Acrophase & $0822 \pm 0454^{\star} \#$ & $0246 \pm 0306$ & $4.97 \pm 2.60$ \\
Peak width & $1312 \pm 0545$ & $1131 \pm 0516$ & $0547 \pm 0400$ \\
Ultradian amplitude & $1.99 \pm 1.08$ & $2.37 \pm 2.20$ & $1355 \pm 0502$ \\
Ultradian harmonic & $3.54 \pm 0.78$ & $3.40 \pm 0.83$ & $3.00 \pm 2.13$ \\
\hline
\end{tabular}

${ }^{*} P<0.05$ compared with phase II, Mardia-Watson-Wheeler $\chi^{2}=6.66, \mathrm{df}=2$.

$\# P<0.10$ compared with phase IV, Mardia-Watson-Wheeler $\chi^{2}=4.92, \mathrm{df}=2$. 


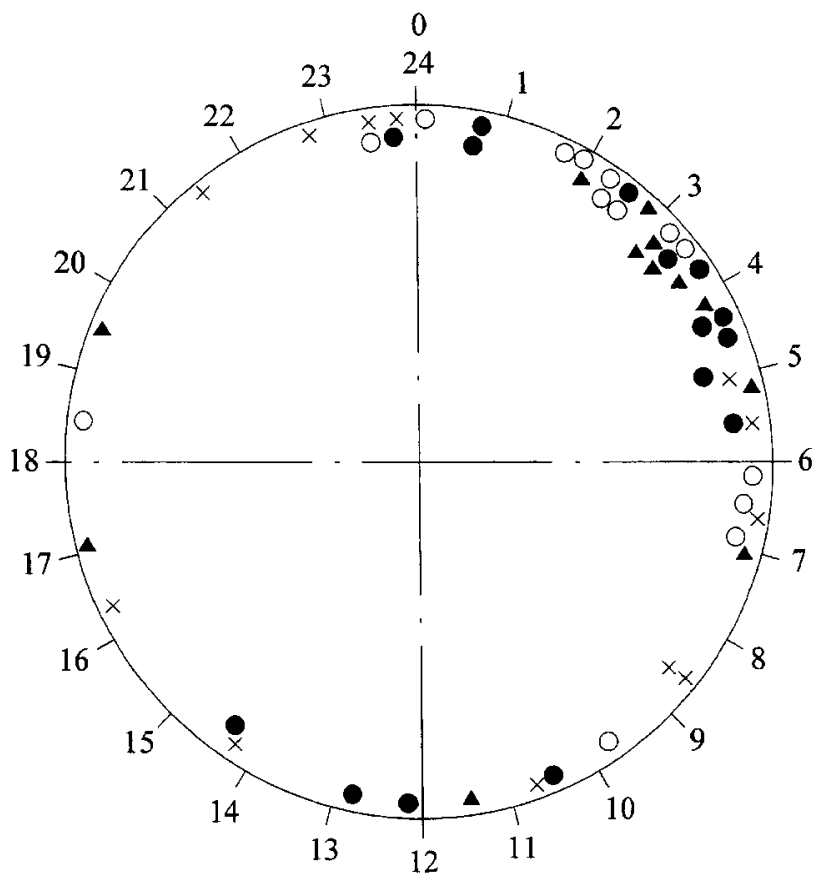

Figure 2 Distribution of acrophase values among different menstrual phases in a total of 53 women. Phase I $(x)$, menstrual phase $(n=12)$; phase II $(\bigcirc)$, late follicular/peri-ovulation phase $(n=14)$; phase III $(\bullet)$, early to mid-luteal phase $(n=15)$; phase IV $(\boldsymbol{\Delta})$, late luteal phase $(n=12)$. Note acrophases were concentrated in the early morning.

estradiol, estimated to represent only about $1-2 \%$ of the total estradiol (33). Our finding that acrophases converge in the early morning is in agreement with the studies of Mitamura et al. (12) and Norjavaara et al. (34) on diurnal rhythms of estradiol. They applied a 24-h blood sampling protocol in children before and during puberty. It was proposed that the increased secretion of estradiol in the early morning was associated with the nocturnal rise in gonadotropin secretion (34). Our finding of a delay in the morning peak time in the menstrual phase deserves further study.

Animal studies have shown that the SCN is the endogenous circadian pacemaker in the brain which plays an important role in the control of hormonal biological rhythms $(17,35)$. Recent studies have shown that the human SCN expresses receptors for estrogen, androgen and progesterone $(18,36)$. Thus, although a peripheral circadian pacemaker rhythmicity, e.g. one originating in the ovaries, cannot be excluded, the diurnal rhythm of estrogens, as found in the present study, is most likely driven by the SCN, and is integrated with signals from the hypothalamic $\mathrm{GnRH}$ and the pituitary gonadotropin secretion profiles $(11-16)$.

The study of estrogen requires full appreciation of its oscillatory dynamics, and the value of evaluating a person's estrogen levels based on a single or just a few time-points is quite limited.

\section{Acknowledgements}

The salivary estradiol data would not have been possible without the help of the volunteers in Hefei. Our appreciation is expressed to Xiao-Hong Chu for help in the salivary estradiol RIA measurement, to Yan-li Wang, Yue-ju Wang and Xin-Hui Wang for assistance, and to Frank PM Kruijver and Professor DF Swaab (Netherlands Institute for Brain Research, Amsterdam) for offering comments. This work was financially supported by the National Nature Science Foundation of China C030313, KNAW 01CDP019. EVS was supported by NIBR Amsterdam, ZON-MW The Hague (project 28-3003), NWO The Hague (projects SOW 014-90-001 and Vernieuwingsimpuls), Stichting Centraal Fonds RVVZ and the Japan Foundation for Aging and Health.

\section{References}

1 Shughrue PJ \& Merchenthaler I. Estrogen is more than just a "sex hormone': novel sites for estrogen action in the hippocampus and cerebral cortex. Frontiers in Neuroendocrinology 200021 95-101.

2 Farhat MY, Lavigne MC \& Ramwell PW. The vascular protective effects of estrogen. FASEB Journal 199610 615-624.

3 Turner RT, Riggs BL \& Spelsberg TC. Skeletal effects of estrogen. Endocrine Reviews 199415 275-296.

4 Bilezikian JP. The role of estrogens in male skeletal development. Reproduction, Fertility and Development 20013 253-259.

5 Swaab DF \& Hofman MA. Sexual differentiation of the human hypothalamus in relation to gender and sexual orientation. Trends in Neurosciences $1995 \mathbf{1 8} 264-270$.

6 Luine VN, Richards ST, Wu VY \& Beck KD. Estradiol enhances learning and memory task and affects levels of monoaminergic neurotransmitters. Hormones and Behavior 199834 149-162.

7 McEwen BS. Clinical review 108. The molecular and neuroanatomical basis for estrogen effects in the central nervous system. Journal of Clinical Endocrinology and Metabolism $1999 \quad \mathbf{8 4}$ 1790-1797.

8 Novaes C, Almeida OP \& Melo NR. Mental health among perimenopausal women attending a menopause clinic. Possible association with premenstrual syndrome? Climacteric $19981264-270$.

9 Ahckas A, Kaukoranta J \& Alto M. Effect of oestradiol on postpartum depression. Psychopharmacology 1999146 108-110.

10 Carlson LE, Sherwin BB \& Chertkow HM. Relationship between mood and estradiol $\left(E_{2}\right)$ levels in Alzheimer's disease (AD) patients. Journal of Gerontology: Psychological Sciences $2000 \mathbf{5 5}$ $47-53$.

11 Lenton EA, Cooke ID, Sampson GA \& Sexton L. Oestradiol secretion in men and pre-menopausal women. Clinical Endocrinology $1978937-47$.

12 Mitamura R, Yano K, Suzuki N, Ito Y, Makita Y \& Okuno A. Diurnal rhythms of luteinizing hormone, follicle-stimulating hormone, testosterone, and estradiol secretion before the onset of female puberty in short children. Journal of Clinical Endocrinology and Metabolism 200085 1074-1080.

13 Challis JR, Patrick JE, Campbell K, Natale R \& Richardson B. Diurnal changes in maternal plasma oestrone and oestradiol at 30 to 31,34 to 35 and 38 to 39 weeks gestational age. British Journal of Obstetrics and Gynaecology 198087 983-988.

14 McGregor JA, Hastings C, Roberts T \& Barrett J. Diurnal variation in saliva estriol level during pregnancy: a pilot study. American Journal of Obstetrics and Gynecology 1999180 223-225.

15 Panico S, Pisani P, Muti P, Recchione C, Cavalleri A, Totis A \& Berrino F. Diurnal variation of testosterone and estradiol: a 
source of bias in comparative studies on breast cancer. Journal of Endocrinological Investigation 199013 423-426.

16 Knobil E. The neuroendocrine control of the menstrual cycle. Recent Progress in Hormone Research 198036 53-88.

17 van der Beek EM, Wiegant VM, van der Donk HA, van den Hurk R \& Buijs RM. Lesions of the suprachiasmatic nucleus indicate the presence of a direct vasoactive intestinal polypeptide-containing projection to gonadotrophin-releasing hormone neurons in the female rat. Journal of Neuroendocrinology 19935 137-144.

18 Kruijver FPM \& Swaab DF. Sex hormone receptors are present in the human suprachiasmatic nucleus. Neuroendocrinology 2002 $75296-365$.

19 Walker RF, Read GF, Wilson DW, Riad-Fahmy D \& Griffiths K. Chronobiology in laboratory medicine: principles and clinical applications illustrated from measurements of neutral steroids in saliva. Progress in Clinical and Biological Research 1990341 105-117.

20 Luo LL. In Infertility and Sterility, edn 1, pp 19, 732. Ed. Luo Li-Lan. Beijing: People Hygiene Press, 1999 [In Chinese].

21 Scheer FA \& Buijs RM. Light affects morning salivary cortisol in humans. Journal of Clinical Endocrinology and Metabolism 1999 84 3395-3398.

22 Shirtcliff EA, Granger DA, Schwartz EB, Curran MJ, Booth A \& Overman WH. Assessing estradiol in biobehavioral studies using saliva and blood spots: simple radioimmunoassay protocols, reliability, and comparative validity. Hormones and Behavior 200038 137- 147 .

23 Halberg F, Johnson EA, Nelson W, Runge W \& Sothern R. Autorhythmometry procedures for physiological self-measurements and their analysis. Physiology Teacher 19721 1-11.

24 Horn R. Statistical methods for model discrimination. Biophysical Journal $198751255-263$.

25 Batschelet E. Circular Statistics in Biology, pp 169-177. New York, NY: Academic Press Inc, 1981.

26 Czeisler CA, Duffy JF, Shanahan TL, Brown EN, Mitchell JF, Rimmer DW et al. Stability, precision and near-24 hour period of the human circadian pacemaker. Science $1999 \quad \mathbf{2 8 4}$ $2177-2181$
27 Minors DS \& Waterhouse JM. The use of constant-routines in unmasking the endogenous component of the human diurnal rhythms. Chronobiology International 19841 205-216.

28 Stern K \& McClintock M. Regulation of ovulation by human pheromones. Nature 1998392 177-179.

29 Riad-Fahmy D, Read GF, Walker RF, Walker SM \& Griffiths K. Determination of ovarian steroid hormone levels in saliva: an overview. Journal of Reproductive Medicine 198732 254-272.

30 Vining RF, McGinley RA \& Symons RG. Hormones in saliva: mode of entry and consequent implications for clinical interpretation. Clinical Chemistry $1983291752-1756$.

31 Choe JK, Khan-Dawood FS \& Dawood MY. Progesterone and estradiol in the saliva and plasma during the menstrual cycle. American Journal of Obstetrics and Gynecology $1983147557-562$.

$32 \mathrm{Lu}$ Y, Bentley GR, Gann PH, Hodges KR \& Chatterton RT. Salivary estradiol and progesterone levels in conception and nonconception cycles in women: evaluation of a new assay for salivary estradiol. Fertility and Sterility 199971 863-868.

33 Tamate K, Charleton M, Gosling JP, Egan D, Ishikawa M, Fottrell PF et al. Direct colorimetric monoclonal antibody enzyme immunoassay for estradiol-17 beta in saliva. Clinical Chemistry 1997 43 1159-1164.

34 Norjavaara E, Ankarberg C \& Albertsson-Wikland K. Diurnal rhythm of 17-beta-estradiol secretion throughout pubertal development in healthy girls: evaluation by a sensitive radioimmunoassay. Journal of Clinical Endocrinology and Metabolism 199681 4095-4102.

35 Buijs RM \& Kalsbeek A. Hypothalamic interaction of central and peripheral clocks. Nature Reviews Neuroscience 20012 521-526.

36 Fernandez-Guasti A, Kruijver FP, Fodor M \& Swaab DF. Sex differences in the distribution of androgen receptors in the human hypothalamus. Journal of Comparative Neurology $2000 \mathbf{4 2 5}$ 422-435.

Received 5 September 2002

Accepted 15 November 2002 